\title{
Unveiling the nature of IGR J16493-4348 with IR spectroscopy (Research Note)
}

\author{
E. Nespoli ${ }^{1}$, J. Fabregat ${ }^{1}$, and R. E. Mennickent ${ }^{2}$ \\ 1 Observatorio Astronómico de la Universidad de Valencia, Calle Catedrático Agustín Escardino 7, 46980 Paterna, Valencia, Spain \\ e-mail: elisa.nespoli@uv.es \\ 2 Departamento de Astronomía, Universidad de Concepción, Casilla 160-C, Concepción, Chile
}

Received 3 March 2010 / Accepted 8 April 2010

\begin{abstract}
Context. The International Gamma-Ray Astrophysics Laboratory (INTEGRAL) is discovering a large number of new hard X-ray sources, many of them being HMXBs. The identification and spectral characterization of their optical/infrared counterparts is a necessary step in undertaking a detailed study of these systems.

Aims. In a previous paper, we presented spectral analyses and classifications of six newly discovered INTEGRAL sources. In this paper, we extend the analysis to IGR J16493-4348.

Methods. We used the ESO/VLT ISAAC spectrograph to observe the proposed IR counterpart to the source, obtaining a $K_{\mathrm{s}}$ mediumresolution spectrum $(R=500)$ with a signal-to-noise ratio $(S / N) \gtrsim 150$. We classified the source by comparing with published atlases. Results. We spectrally classified the source as a B0.5-1 supergiant and estimated its interstellar extinction. We compared the extinction derived from X-ray data with effective interstellar extinction obtained from our data, discussing the absorption component associated with the circumstellar environment.
\end{abstract}

Key words. X-rays: binaries - binaries: symbiotic - accretion, accretion disks - infrared: stars

\section{Introduction}

High mass X-ray binaries (HMXBs) are composed of an earlytype massive star and an accreting compact object, a neutron star, or a black hole. The majority of known systems are Be/X-ray binaries (BeXRBs), consisting of a neutron star accreting matter from the circumstellar equatorial disk of a Be star. Most of them are transient, exhibiting short and bright outbursts in the X-ray band. In the second major class of HMXBs, the supergiant X-ray binaries (SGXRBs), the compact object accretes matter from an early supergiant star through its radially outflowing stellar wind. As a consequence, most of SGXRBs are persistent systems, with $L_{\mathrm{X}} \sim 10^{36} \mathrm{erg} \mathrm{s}^{-1}$.

The INTEGRAL survey of the Galactic plane and central regions is helping to substantially improve our knowledge of Galactic X-ray binaries (Bird et al. 2007, 2010). A large fraction of the newly discovered sources are heavily obscured supergiant massive X-ray binaries (first suggested by Revnivtsev et al. 2003), exhibiting much larger column densities $\left(N_{\mathrm{H}} \gtrsim\right.$ $10^{23} \mathrm{~cm}^{-2}$ ) than expected along the line of sight (see Kuulkers 2005). These sources were missed by previous high-energy missions, whose onboard instruments were sensitive to a softer energy range. Optical counterparts to these obscured sources are also barely detectable because of the high interstellar extinction, $A_{V}$ being in excess of up to $\sim 20 \mathrm{mag}$.

In this context, infrared spectroscopy is an important tool for characterizing these systems (see also Nespoli et al. 2008b, hereafter referred to as Paper I). With high-energy data, it helps us to identify the HMXB subclass the sources belong to and

* Based on observations collected at the European Southern Observatory, Chile (Programme ID 079.D-0668-B). the mass-transfer process of the system, providing information about the intrinsic physics of the X-ray binary.

In Paper I, we presented IR spectroscopy of six INTEGRAL sources, classifying their counterparts and estimating both their distance and interstellar extinction. In this paper, we extend the analysis including one more INTEGRAL source, IGR J164934348 , located in the direction of the Norma-arm tangent region.

The source was discovered by Grebenev et al. (2005). Subsequent RXTE observations by Markwardt et al. (2005) found that the mean spectrum is consistent with a heavily absorbed power law with $N_{\mathrm{H}} \sim 10^{23} \mathrm{~cm}^{-2}$ and a photon index of 1.4. The measured flux was $1.0,1.3$, and $2.1 \times 10^{-11} \mathrm{erg} \mathrm{cm}^{-2} \mathrm{~s}^{-1}$ in the 2-10, 10-20, and 20-40 keV energy bands, respectively. Kuiper et al. (2005) performed Chandra imaging of the field of IGR J16493-4348 for $4.1 \mathrm{ks}$. They detected a single point source within the $2^{\prime}$ error circle of the INTEGRAL source at $\mathrm{RA}=16^{\mathrm{h}} 49^{\mathrm{m}} 26.92^{\mathrm{s}}$, Dec $=-43^{\circ} 49^{\prime} 8.96^{\prime \prime}$, with a $0.6^{\prime \prime}$ error in each coordinate. No spectrum could be extracted from the data and Kuiper et al. (2005) noted that previous measurements of IGR J16493-4348 by RXTE may be contaminated by another X-ray source, 1RXS J164913.6-435527, located $\sim 6.7^{\prime}$ away. They also pointed out that no significant source can be found at the position of the Chandra source in the optical DSS maps, indicating strong absorption in the direction of the source. From INTEGRAL data, Hill et al. (2008) discarded the previously proposed association with the free radio pulsar PSR J16494349; the best-fit model of the X-ray spectrum obtained by the same authors included an absorbed cut-off powerlaw with $N_{\mathrm{H}}=5.4 \times 10^{22} \mathrm{~cm}^{-2}$ and $\Gamma=0.6$. From Suzaku data, Morris et al. (2009) obtained $N_{\mathrm{H}}=2.6_{-0.8}^{+0.9} \times 10^{23} \mathrm{~cm}^{-2}$ and $\Gamma=2.4$. Although having different parameters, both results are consistent with an accreting neutron star. 
A\&A 516, A106 (2010)

Table 1. VLT/ISAAC observation log.

\begin{tabular}{lc}
\hline \hline Start time (UT) & 2007-04-05 09:38 \\
Exp. time (s) & 360 \\
$S / N$ & 150 \\
$R(\lambda / \Delta \lambda)$ & 500 \\
IR counterpart & 2MASS J16492695-4349090 \\
$K$ mag & 11.9 \\
Reference & Kuiper et al. (2005) \\
\hline
\end{tabular}

The infrared counterpart to the source was proposed by Kuiper et al. (2005), who reported a single 2MASS source, 2MASS J16492695-4349090, compatible with both the Chandra and Swift/XRT positions. Kuiper et al. (2005) observed the source in the $K_{\mathrm{s}}$ band and found a magnitude of 12, consistent with the 2MASS magnitude. No optical/IR spectra of the counterpart are available, and the nature of the system, although its position and X-ray behavior suggest that it is a HMXB system, remains unproven.

In the next section, we describe the observations and data reduction. In Sect. 3, we present the obtained spectrum, analyze its features to propose a classification, and calculate the interstellar hydrogen column density. In Sect. 4, we discuss our results, before concluding. Preliminary results of our data analysis were published in Nespoli et al. (2008a).

\section{Observations and data analysis}

Data were obtained in service mode on 2007 April 5 with the ISAAC spectrograph (Moorwood et al. 1998) on UT1 at ESO/Paranal observatory. The sky was clear during the observations, the seeing was $\leq 1.4^{\prime \prime}$, and the target was observed at airmass 1.09. Data were taken in the short-wavelength, low-resolution mode, in the $K$ band, with a pixel scale of $0.147^{\prime \prime} /$ pixel and a resolution of 500. Table 1 presents the observation log, including the signal-to-noise ratio $(S / N)$ achieved per resolution element. Typical on-source integration times for standard stars were between 6 and $10 \mathrm{~s}$.

Data reduction was performed using the $\mathrm{IRAF}^{1}$ package, following the standard procedures for IR spectra, described in detail in Paper I. Removal of atmospheric features was accomplished by employing a G2 V and a A3 V spectrum, following a strategy similar to that outlined by Clark \& Steele (2000). For a detailed description of the correction procedure, we refer to Paper I. Between the standard telluric spectra and the scientific one, efforts were made to ensure differences of no more $\sim 0.01$ in the airmass.

\section{Results}

We present our spectral classification and analysis results for IGR J16493-434. Our data is the first available spectrum of this source. The analysis will be qualitative, based on comparison with available NIR spectral atlases (Hanson et al. 1996, 2005).

The processed $K$-band spectrum is shown in Fig. 1, while its most significant features are reported in Table 2.

\footnotetext{
1 IRAF is distributed by the National Optical Astronomy Observatories which is operated by the Association of Universities for Research in Astronomy, Inc. under contract with the National Science Foundation.
}

Table 2. $K$-band line identifications.

\begin{tabular}{|c|c|c|c|}
\hline Feature & Transition & Wavel. [̊] & $E W[\AA]$ \\
\hline $\mathrm{He} \mathrm{I}$ & $\left(2 \mathrm{~s}^{1} \mathrm{~S}-2 \mathrm{p}^{1} \mathrm{P}^{\circ}\right), 20581 \AA$ & 20571 & $-2.7 \pm 0.4$ \\
\hline \multirow[t]{2}{*}{$\mathrm{He} \mathrm{I}$} & $\left(3 \mathrm{p}^{3} \mathrm{P}^{\circ}-4 \mathrm{~s}^{3} \mathrm{~S}\right), 21120 \AA$ & & \\
\hline & $+\left(3 \mathrm{p}^{1} \mathrm{P}^{\circ}-4 \mathrm{~s}^{1} \mathrm{~S}\right), 21130 \AA$ & 21112 & $2.0 \pm 0.4$ \\
\hline $\operatorname{Br} \gamma$ & $\left(4^{2} \mathrm{~F}^{\circ}-7^{2} \mathrm{G}\right), 21661$ & 21655 & $2.7 \pm 0.2$ \\
\hline
\end{tabular}

Errors in the equivalent widths were calculated as deviations from the mean values, measured using different estimates of the continuum level. Some of the identified features exhibit a 5-10 A displacement with respect to the nominal values, which is consistent with the instrumental resolution.

Although it has been pointed out (Hanson et al. 1996) that equivalent widths may vary between stars of the same spectral type, we report our measurements for completeness.

\subsection{Spectral analysis and classification}

The spectrum exhibits a strong emission He I $20581 \AA$ A line, He I $21126 \AA$ in absorption, and a relatively strong absorption $\mathrm{Br} \gamma$ $21661 \AA ̊$ line. We also tentatively detected $\mathrm{Br} \gamma$ in faint emission, which might be a signature of an accretion disk around the compact object. However, the detection is very weak, and we cannot exclude it being a residual artifact from the telluric-line removal procedure, which in this region is very critical. The atomic transitions detected are the typical ones of OB stellar spectra. In particular, He I $20581 \AA$ is a prominent feature for supergiant stars and is observed in emission in B type supergiants. The He I $21126 \AA$ line is present in late O-early B spectra. By comparing the relative strength of the identified lines with those from the atlas of Hanson et al. (1996), we estimated the spectral type to be B0.5-1 Ia-Ib. With information provided by X-ray data (Hill et al. 2008), our results thus permit us to classify the system as a neutron-star SGXRB.

\subsection{Reddening and distance estimation}

As in Paper I, we proceeded to calculate the extinction and the distance to characterize the system.

From the proposed spectral classification, we obtained the intrinsic colors $(J-K)_{0}$ from Wegner (1994). Apparent colors were calculated from 2 MASS photometry, properly transformed by means of the formula from Carpenter $(2001)^{2}$ to the Bessell \& Brett (1988) homogenized photometric system to estimate the infrared color excess $E(J-K)$. We assumed the mean extinction law $\left(R_{V}=3.1\right)$, from which $A_{V} / E(J-K)=5.82 \pm 0.10$ (Rieke $\&$ Lebofsky 1985), obtaining the total measured visual extinction $A_{V}$, and the corresponding hydrogen column density value from $N_{\mathrm{H}} / A_{V}=1.79 \pm 0.03 \times 10^{21}$ atoms cm $\mathrm{cmag}^{-1}$ (Predehl $\&$ Schmitt 1995). We were thus able to compare the measured interstellar value of $N_{\mathrm{H}}$ with that inferred from X-ray data. In our calculation, we estimated errors by means of error propagation. The error in the final value of $N_{\mathrm{H}}$ is mainly caused by errors in the infrared colors and the transformation between the two photometric systems. In Table 3, we present the results of our calculations, namely the spectral classification of the IR source, the intrinsic infrared colors, 2MASS photometry, the infrared

\footnotetext{
2 In its updated version at http://www.astro.caltech.edu/ jmc/2mass/v3/transformations/
} 


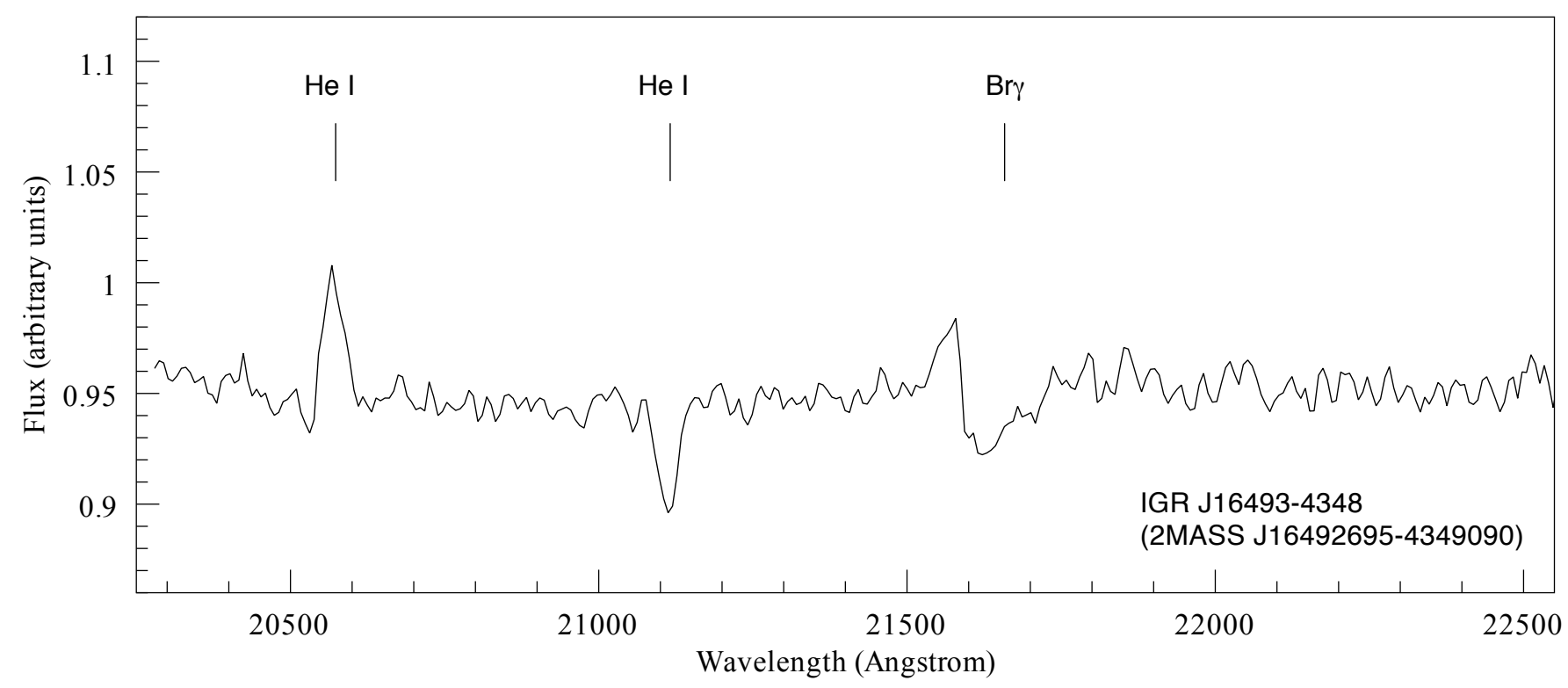

Fig. 1. $K_{\mathrm{s}}$ spectrum for 2MASS J16492695-4349090, the infrared counterpart of IGR J16493-4348. The positions of identified spectral features are indicated by solid lines.

Table 3. Results from reddening estimation.

\begin{tabular}{lr}
\hline \hline Spectral type & B0.5-1 Ia-Ib \\
$(J-K)_{0}(\mathrm{mag})$ & -0.12 \\
$(J-K)_{2 \mathrm{MASS}}(\mathrm{mag})$ & 2.66 \\
$E(J-K)(\mathrm{mag})$ & 2.81 \\
$N_{\mathrm{H}}$ from X-ray data $\left(10^{22} \mathrm{~cm}^{-2}\right)$ & $26_{-7.9}^{+9.4}$ \\
Interstellar $N_{\mathrm{H}}\left(10^{22} \mathrm{~cm}^{-2}\right)$ & $2.92 \pm 1.96$ \\
\hline
\end{tabular}

excess, the hydrogen column density obtained from X-ray published measurements (most recent results), and the effective interstellar column density obtained from our work.

We also estimated the distance to the source, by means of the relation $M_{K}=K+5-5 \log d-A_{K}$, employing intrinsic colors and 2MASS magnitudes (see Paper I for procedures and references). For this system, the huge uncertainty in the intrinsic colors directly affects our estimate of distance, which may have any values in the range $6-26 \mathrm{kpc}$. This range is too broad to allow us to determine the precise location of the source in the Galaxy, and in particular to confirm its association with any Galactic spiral structure. Our distance measurement is thus not shown in Table 3.

\section{Discussion and conclusions}

Using $K$-band spectroscopy, we classified the counterpart to IGR J16493-4348 by comparison with published atlases. We found that the system has a supergiant companion, estimating its type to be B0.5-1 Ia-Ib. Combined with information from X-ray data, our results permit us to classify the system as a SGXRB.

This work allowed us to calculate the extinction from IR data. Previous works have pointed out (see Kuulkers 2005; Chaty et al. 2008) that INTEGRAL is detecting a new class of highly obscured supergiant HMXBs. The origin and position (around the compact object only, or enveloping the entire system) of the absorbing material remain unclear, and only multiwavelength studies can help us to address the problem, by distinguishing between the absorption in X-ray and in the IR/optical bands.

We calculated the effective interstellar extinction $A_{V}$ and converted it into a hydrogen column density of $N_{\mathrm{H}}=2.92 \pm$ $1.96 \times 10^{22}$. This value is compatible with estimates of the weighted average neutral hydrogen density in a cone of radius $1^{\circ}$ along the line of sight of IGR J16493-4348 ( 1.4$1.8 \times 10^{22} \mathrm{~cm}^{-2}$, Kalberla et al. 2005; Dickey \& Lockman 1990). Our results were compared with the values obtained from X-ray data. If the two derived values were compatible within their corresponding errors, we would have to consider two possible scenarios: either the source of absorption is just the interstellar medium, or there is a contribution from an extensive envelope around the whole binary system, if the extinction is systematically higher by some orders of magnitude than the estimated interstellar value. In contrast, if the reddening (measured from the IR colors) were low compared to the measured $N_{\mathrm{H}}$ from X-rays, this would imply that there is an additional source of extinction, which only affects the compact object in which the X-ray emission originates and can be assumed to be the absorbing material around it.

In our case, the extinction measured at high energy was found to be one order of magnitude higher than that obtained from IR data, which indicates that the material absorbing in the X-ray is concentrated around the neutron star. The same results were found in Paper I for the following systems: IGR J16465-4507, IGR J16479-4514, AX J1841.0-0536, and IGR $\mathrm{J} 19140+0951$.

The so-called highly absorbed IGR sources are usually identified to be those for which the measured $N_{\mathrm{H}}$ is $\gtrsim 10^{23} \mathrm{~cm}^{-2}$, i.e., one to two orders of magnitude higher than the assumed Galactic value of $\sim 10^{22} \mathrm{~cm}^{-2}$ (Kuulkers 2005). As in Paper I, the comparison of X-ray data, which are sensitive to the absorption caused by the environment of the compact object, with infrared data, which indicate in general only the radiation absorbed by the interstellar medium, was a powerful, alternative criterion for identifying this class of highly absorbed sources. 


\section{Conclusions}

Using $K$-band spectroscopy, we have extended the analysis presented in Paper I to another INTEGRAL source, IGR J164934348, which is part of the new class of highly-obscured SGXRBs. We have presented the first available IR spectrum of the counterpart and found that:

- The proposed optical counterpart was confirmed and classified as B0.5-1 Ia-Ib.

- The system was thus classified as a SGXRB.

- The comparison between $N_{\mathrm{H}}$ obtained from X-ray data and the interstellar extinction from our data showed the presence of an absorbing envelope, tightly confined to the vicinity of the compact object.

Acknowledgements. The work of E.N. and J.F. is supported by the Spanish Ministerio de Educación y Ciencia, and FEDER, under contract AYA 2007 62487. This work has been partly supported by the Generalitat Valenciana project of excellence PROMETEO/2009/064. E.N. acknowledges a "V Segles" research grant from the University of Valencia. R.E.M. acknowledges support by Fondecyt 1070705, the Chilean Center for Astrophysics FONDAP 15010003 and the BASAL Centro de Astrofísica y Tecnologías Afines (CATA) PFB-06/2007.

\section{References}

Bessell, M. S., \& Brett, J. M. 1988, PASP, 100, 1134
Bird, A. J., Bazzano, A., Bassani, L., et al. 2010, ApJS, 186, 1 Bird, A. J., Malizia, A., Bazzano, A., et al. 2007, ApJS, 170, 175 Carpenter, J. M. 2001, AJ, 121, 2851

Chaty, S., Rahoui, F., Foellmi, C., et al. 2008, A\&A 484, 783

Clark, J. S., \& Steele, I. A. 2000, A\&AS, 141, 65

Dickey, J. M., \& Lockman, F. J. 1990, ARA\&A, 28, 215

Grebenev, S. A., Bird, A. J., Molkov, S. V., et al. 2005, The Astronomer's Telegram, 457, 1

Hanson, M. M., Conti, P. S., \& Rieke, M. J. 1996, ApJS, 107, 281

Hanson, M. M., Kudritzki, R.-P., Kenworthy, M. A., Puls, J., \& Tokunaga, A. T. 2005, ApJS, 161, 154

Hill, A. B., Dean, A. J., Landi, R., et al. 2008, MNRAS, 385, 423

Kalberla, P. M. W., Burton, W. B., Hartmann, D., et al. 2005, A\&A, 440, 775

Kuiper, L., Jonker, P., Hermsen, W., \& O'Brien, K. 2005, The Astronomer's Telegram, 654, 1

Kuulkers, E. 2005, in Interacting Binaries: Accretion, Evolution, and Outcomes, ed. L. Burderi, L. A. Antonelli, F. D’Antona, T. di Salvo, G. L. Israel, L. Piersanti, A. Tornambè, \& O. Straniero, AIP Conf. Ser., 797, 402

Markwardt, C. B., Swank, J. H., \& Smith, E. 2005, The Astronomer's Telegram, 465,1

Moorwood, A., Cuby, J.-G., Biereichel, P., et al. 1998, The Messenger, 94, 7 Morris, D. C., Smith, R. K., Markwardt, C. B., et al. 2009, ApJ, 699, 892

Nespoli, E., Fabregat, J., \& Mennickent, R. E. 2008a, The Astronomer's Telegram, 1396, 1

Nespoli, E., Fabregat, J., \& Mennickent, R. E. 2008b, A\&A, 486, 911

Predehl, P., \& Schmitt, J. H. M. M. 1995, A\&A, 293, 889

Revnivtsev, M. G., Sazonov, S. Y., Gilfanov, M. R., \& Sunyaev, R. A. 2003, Astron. Lett., 29, 587

Rieke, G. H., \& Lebofsky, M. J. 1985, ApJ, 288, 618

Wegner, W. 1994, MNRAS, 270, 229 